\title{
INFLUÊNCIA DE FACTORES SOCIOCULTURAIS E DA DIMEN- SÃO INDEPENDÊNCIA-INTERDEPENDÊNCIA NO FOCO DA ANSIEDADE SOCIAL
}

\author{
José Paulo Mota ${ }^{1}$
}

Resumo: Neste artigo de revisão, procura-se salientar a importância e potencial efeito de determinantes socioculturais e da dimensão independência-interdependência na forma de manifestação subjectiva da ansiedade social. Para cumprir esse objectivo, são revistos os sintomas geralmente associados à ansiedade social desadaptativa tal como descritos no DSM-IV-TR e no CID-10 (associados portanto à cultura ocidental, particularmente europeia e norte-americana moderna), procura-se analisar relatos bibliográficos de vivências desse tipo de ansiedade em outras culturas (nomeadamente em culturas asiáticas, com especial ênfase na cultura japonesa), e faz-se uma revisão bibliográfica de literatura relativa a esta temática. Formam-se assim as bases para discussão do tópico proposto, e para o levantamento de questões relevantes para a investigação futura e para a prática clínica ${ }^{2}$.

Palavras-Chave: Ansiedade social, Taijin-kyofu-sho, variáveis socioculturais, dimensão independência-interdependência, embaraço, antropologia cultural.

Influence of socio-cultural factors and the independence-interdependence dimension on the focus of Social Anxiety (Abstract): The objective of this review article is the highlighting of the importance and potential effect of socio-cultural variables and the independence-interdependence dimension on how social anxiety will manifest itself. To accomplish that goal, a review is presented of the symptoms commonly associated with maladaptive social anxiety as described in DSM-IV-TR and CID-10 (associated thus with western culture, especially contemporary European and North-American). In addition, an analysis of bibliographical accounts of such kinds of anxiety as experienced in other cultures (Asian cultures in particular, with special emphasis on Japanese culture), and a bibliographical review of the literature concerning this topic are presented. These form the basis for the discussion of

\footnotetext{
${ }^{1}$ Licenciado e Mestre em Psicologia pela Faculdade de Psicologia e de Ciências da Educação da Universidade do Porto. Membro do Centro de Desenvolvimento Vocacional e Formação ao Longo da Vida (Centro de Psicologia da Universidade do Porto). Email: jpmota_05@yahoo.com.

${ }^{2}$ Abreviaturas Utilizadas: Taijin-kyofu-sho (TKS) / Manual de Diagnóstico e Estatística das Perturbações Mentais (DSM) / Classificação Internacional das Doenças e Problemas de Saúde Relacionados (CID).
} 
the proposed topic, and for the raising of questions relevant to future investigation and clinical practice.

Keywords: Social anxiety, Taijin-kyofu-sho, socio-cultural variables, independence-interdependence dimension, embarassment, cultural anthropology.

\section{A vivência da ansiedade social}

\subsection{A ansiedade social na cultura ocidental}

Apesar de já existirem referências médicas relativas à ansiedade social desde o tempo de Hipócrates, o conceito contemporâneo deste tipo de ansiedade foi inicialmente formulado por Marks e Gelder (1966), tendo sido posteriormente desenvolvido no seguimento da sua inclusão no DSM-III em 1980 (Heimberg \& Becker, 2002). Refere-se, em termos gerais, ao aumento de ansiedade num indivíduo quando este se encontra em situações de interacção social, seja de uma forma generalizada ou em situações específicas. Pode variar num continuum entre uma leve ansiedade (frequentemente adaptativa e promotora da acção), até um extremo de fobia social generalizada (forte ansiedade numa variedade de situações sociais, frequentemente incapacitante), induzindo mecanismos de defesa como a fuga ou o evitamento social. Aos casos mais graves de ansiedade social está geralmente associado o abandono precoce da vida escolar, dependência económica, baixo rendimento e instabilidade no emprego, dificuldades afectivas, e baixo suporte social (Gouveia, 2000). Butler (1999, p. 4) descreve a ansiedade social como "o medo, nervosismo, e apreensão que muitas pessoas sentem na sua relação com outras pessoas", acrescentando que "afecta as pessoas quando estas pensam estar a fazer algo que possa ser humilhante ou embaraçoso (...), fazendo-as achar que os outros a estão a julgar, de forma negativa, por algo que disse ou fez (...), inibindo a pessoa e tornando-a auto-consciente". Para Gouveia (2000), a ansiedade social é uma experiência humana comum, intimamente relacionada com a estrutura social de grupo e sua organização hierárquica. Este autor refere ainda (citando Clark \& Wells, 1995), que os esquemas cognitivos de incompetência social associados a esta perturbação são instáveis, ou seja, fora das situações sociais em que podem ser avaliados e que consideram ameaçadoras, os fóbicos sociais possuem uma visão mais favorável de si próprios.

A Classificação Internacional das Doenças e Problemas de Saúde Relacionados, na sua classificação de perturbações mentais e de comportamento (OMS, 1993), e o Manual de Diagnóstico e Estatística das Perturbações Mentais - $4^{a}$ Edição - Texto Revisto (APA, 2002), enquanto principais manuais de referência no que respeita a perturbações mentais na cultura 
ocidental, apresentam os seguintes critérios de diagnóstico de ansiedade social desadaptativa (Fobia Social):

a) Medo intenso e persistente de uma ou mais situações sociais nas quais o indivíduo está exposto a pessoas desconhecidas ou ao possível escrutínio de outros, receando exibir comportamentos embaraçosos ou sintomas de ansiedade (havendo um reconhecimento por parte do indivíduo de que o medo é excessivo ou irracional);

b) Frequência elevada da resposta de ansiedade face à exposição a situações sociais receadas (podendo predispor situacionalmente a um ataque de pânico), em que a ansiedade sentida não é devida a efeitos fisiológicos duma substância ou a uma condição física geral, e não é melhor explicada por outra perturbação mental;

c) Pode apresentar-se na forma de rubor, tremores ou náuseas, e pode levar ao evitamento das situações sociais ou de desempenho receadas, ou a um confronto com as mesmas feito com intensa ansiedade ou desconforto;

d) $\mathrm{O}$ evitamento, ansiedade antecipatória ou desconforto interferem significativamente no funcionamento normal do indivíduo;

e) Divide-se em dois sub-tipos possíveis: Fobia Social Especifica (ocorrendo em uma ou duas situações sociais específicas) e Fobia Social Generalizada (manifestando-se na maioria das situações sociais), considerando-se geralmente que o segundo sub-tipo afecta mais a qualidade de vida dos indivíduos, dada a variedade de situações abrangidas;

f) O início ocorre frequentemente na adolescência, sendo igualmente comum em homens e mulheres, e está associado a baixa auto-estima e medo de críticas.

Estas constituem as formas predominantes de manifestação da ansiedade social, em culturas ocidentais, embora lhes sejam por vezes apontadas algumas limitações. Gouveia (2000, pp. 22-23) considera que "apesar destes critérios de diagnóstico [DSM-IV] constituírem um avanço no sentido de uma melhor clarificação do quadro clínico da perturbação de ansiedade social e das suas relações com outros distúrbios de eixo I, mantêm-se algumas dificuldades na sua utilização", dando como exemplo central a não-existência de limiares claros acerca do grau de desconforto e de interferência dos sintomas na vida do indivíduo, necessários para um diagnóstico de fobia social. Pelos critérios referidos compreende-se a interacção entre aspectos cognitivos e físicos, surgindo os elementos comportamentais enquanto respostas psicofisiológicas ou mecanismos de defesa perante a 
ansiedade resultante de uma situação de ameaça percebida, não sendo no entanto devidamente equacionadas as influências possíveis de variáveis culturais e sociais nessa interacção.

$\mathrm{Na}$ última década, tem surgido um crescente movimento no sentido de alargar os critérios de diagnóstico psicopatológico em geral, actualizando os sistemas existentes, de forma a ser devidamente considerada a influência de variáveis socioculturais na vivência de perturbações mentais e comportamentais (Kirmayer, 2001). Em concordância com esta orientação, no campo da intervenção psicológica os códigos de ética e conduta internacionais apelam para esta consciência e complemento, no sentido de melhorar o atendimento em contextos multiculturais (Gielen, Draguns \& Fish, 2008). Estas variáveis têm estado progressivamente no centro de vários estudos, dada a crescente aceitação do seu papel fundamental para uma melhor compreensão da vivência psicopatológica e das suas interacções com os diversos contextos de vida do indivíduo. Ora, manifestando-se o tipo de ansiedade aqui discutido exclusivamente em situações de interacção social (real ou percebida), esta perturbação tem necessariamente uma forte componente sociocultural, apenas podendo ser compreendida numa perspectiva biopsicosocial (resultando de uma interacção entre factores psicológicos, físicos e socioculturais). Neste artigo, focar-se-ão essencialmente os aspectos sociais e culturais, mas tendo sempre em mente a sua indissociabilidade dos restantes.

\subsection{Variações culturais na vivência da ansiedade social}

A análise comparativa de manifestações psicológicas e somáticas entre culturas constitui muitas vezes um instrumento privilegiado para a observação dos efeitos da cultura e sociedade sobre a psicopatologia.

No DSM-IV-TR, é feita referência a um conceito denominado "Síndrome Ligada à Cultura", referindo-se a síndromes cuja existência está limitada a culturas específicas. Mais concretamente, é definido como "um padrão localmente específico e recorrente de comportamentos aberrantes e experiências perturbadoras que pode ou não estar associado a uma determinada categoria diagnóstica do DSM-IV", acrescentando que estas "são geralmente limitadas a sociedades específicas ou zonas culturais e são categorias diagnósticas localizadas e folclóricas que enquadram significados culturais para certos conjuntos de experiências e observações repetitivas, padronizadas e perturbadoras" (APA, 2002, p. 898). Por sua vez, no CID-10 (OMS, 1993) é rejeitada a ideia da existência de perturbações com especificidade cultural total, com base na ausência de estudos epidemiológicos que possam sustentar essa afirmação, apontando-se antes para mani- 
festações particulares de ansiedade, depressão e perturbações somatoformes ou de ajustamento.

Dentro da referida classificação especial do DSM-IV-TR faz-se referência a um quadro clínico presente na literatura psiquiátrica japonesa, denominado taijin-kyofu-sho ${ }^{3}$ (TKS), referindo-se a sintomas de medo perante situações de contacto social face-a-face (Gouveia, 2000). Mais concretamente, este pode ser definido como uma forma de ansiedade social, na qual o indivíduo relata um medo de magoar, ofender ou embaraçar outras pessoas, por oposto a embaraçar-se a si próprio ou ser julgado por outrem como socialmente inapto. Este receio tem como base a ideia de que, ao ter esse efeito negativo noutras pessoas, se traz embaraço para o grupo (família, empresa, etc.). Salienta-se assim a possível influência de um modelo de sociedade colectivista neste quadro de ansiedade. Nesta população, a auto-imagem é predominantemente interdependente relativamente ao grupo, ou seja, o indivíduo é definido essencialmente por referência ao seu grupo (Kleinknecht, Dinnel, Kleinknecht, Himura \& Harada, 1997), sendo portanto o embaraço pessoal desvalorizado e o grupal acentuado. Considera-se que existem quatro sub-tipos através dos quais a TKS se pode manifestar: medo de corar (sekimen-kyofu); medo de ter o corpo deformado (shubo-kyofu); medo de contacto ocular directo (jikoshiken-kyofu); e medo de ter um odor corporal ofensivo (jikoshu-kyofu). Tal como no quadro ocidental de ansiedade social, parecem existir comportamentos de segurança associados, tais como o uso de cremes faciais para disfarçar a ruborização, ou o simples evitamento das situações temidas (Gouveia, 2000). Uma classificação alternativa, que ilustra a continuidade dos sintomas que caracterizam a TKS e demonstra que este é um conceito relativamente mais lato que o da ansiedade social, propõe que esta pode ser classificada em quatro sub-tipos: tipo transiente (ansiedade social característica da adolescência), tipo fóbico (correspondente à definição tradicional de ansiedade social), tipo delirante (caracterizada pela obsessão com defeitos corporais e comportamentos imaginados ou exagerados), e perturbação fóbica acompanhada de esquizofrenia.

Como foi anteriormente referido, também esta caracterização da TKS como síndrome culturalmente especifica não é consensual, havendo autores que consideram que esta não é uma patologia específica à cultura do Japão, mas sim algo de mais generalizado e aproximado das categorias principais do DSM-IV-TR, apesar de pouco estudado (Suzuki, Takei, Kawai, Minabe $\&$ Mori, 2003). Estes autores consideram que nenhum dos sub-tipos ini-

\footnotetext{
${ }^{3}$ Significando "taijin" contacto face-a-face entre duas pessoas, "kyofu" medo, e "sho" sintomas; em conjunto, refere-se a sintomas de medo perante situações sociais de contacto face-a-face.
} 
cialmente referidos é culturalmente distinto no Japão, argumentando que o primeiro constitui um sintoma comum de fobia social, que o segundo se enquadra nos critérios para a "Perturbação Dismórfica Corporal" do DSM-IV-TR (APA, 2002), enquanto que os dois restantes não encontram enquadramento nas categorias mais estudadas mas sim em casos descritos em outras referências bibliográficas ocidentais (Pryse-Phillips, 1971; McNally, Cassiday \& Calamari, 1990). Estes dados são confirmados por Kleinknecht e co-autores (1997), que referem relatos de casos de TKS na Coreia, Estados Unidos da América, e em alguns países da Europa (nomeadamente, Reino Unido, Alemanha e Itália). As semelhanças entre Fobia Social e TKS, bem como a presença desta última em outros contextos culturais, são igualmente salientadas por Gouveia (2000), que refere a presença de sintomas de ambos em casos acompanhados na sua própria experiência clínica em contexto cultural português. Segundo Dinnel, Kleinknecht e Tanaka-Matsumi (2002), os sintomas de TKS são tendencialmente relatados por indivíduos que apresentam menores valores de independência, e valores mais altos de interdependência.

Reforçando esta ideia de variações culturais, Seedat e Nagata (2004) destacam a relevância do estudo das taxas de prevalência da ansiedade social em diferentes países, referindo os resultados de estudos baseados nos critérios da terceira edição do DSM que apontaram para uma discrepância elevada entre países orientais (Taiwan e Coreia) e ocidentais (Alemanha, Estados Unidos da América, França e Nova Zelândia), podendo estes últimos apresentar taxas de prevalência ao longo da vida no mínimo quatro vezes superiores (à volta de 0,4 a $0,6 \%$ nos países orientais, e 2,5 a $4,0 \%$ nos países ocidentais). Aquando da reformulação dos critérios de ansiedade social na quarta edição do DSM, no entanto, as diferenças nestas taxas diminuíram, tornando-se mais comparáveis. Para os autores, as causas dessa discrepância podem ser atribuídas a diferenças de metodologia, de hábitos sociais, e taxas de resposta da população-alvo, e também ao facto da formulação da perturbação de ansiedade social ser de origem ocidental. Este último factor é apontado como relevante, podendo resultar na invisibilidade de sintomas culturalmente específicos potencialmente relevantes, e consequentemente levar a avaliações de valores de prevalência erroneamente reduzidas.

Revistas as particularidades da vivência de duas culturas distintas relativamente ao que se considera ser o mesmo fenómeno subjacente, a ansiedade social, conclui-se que ambos os quadros clínicos podem porventura ser equiparados, tendo em conta aspectos de co-morbilidade, e chamando a atenção para variações de ordem cultural (e individual) determinantes na forma como cada perturbação é vivenciada. 


\section{Influências culturais e pessoais na vivência da ansiedade social}

Pela utilização do termo "factores socioculturais", pretende-se designar factores derivados da interacção entre uma determinada cultura e uma sociedade específica. Para Kluckhohn (1954, citado por Neto, 2003), a cultura está para a sociedade como a memória está para os indivíduos, apontando para uma concepção de sociedade indissociável da sua cultura de origem e funcionando a cultura como o ponto de referência pelo qual uma sociedade define as suas regras e vivências (analogamente, a personalidade de um indivíduo é influenciada pelas suas vivências passadas). Segundo este autor, na cultura de origem estarão incluídas influências sobre a forma como as pessoas percepcionam o seu meio, a si próprias, e juízos sobre o que é o mundo e como as pessoas se deveriam comportar. Para Kirmayer (2001), a cultura exerce um papel determinante em vários aspectos da vida humana, inclusivamente na psicopatologia, nas suas manifestações, e no seu tratamento (tanto do ponto de vista do sujeito que sofre como do terapeuta). Acrescenta ainda que a visão do termo "cultura" se refere hoje em dia não a algo estático, mas a um conjunto de construções temporárias e em permanente mudança, que emergem das interacções entre indivíduo, comunidade, e práticas ideológicas e institucionais. Significa isto que as influências da cultura de origem de um indivíduo não se manifestam de forma pura, mas sim em interacção com diversos factores de várias ordens, gerando uma variabilidade intra-grupal que não pode ser ignorada por profissionais que exercem a sua actividade em contextos multiculturais.

\section{1 - Modelo de sociedade e a variável independência-interdepen- dência}

Ao discutir os principais elementos que caracterizam e distinguem as culturas ocidental e oriental, deve-se ter em conta que essa não representa de forma alguma uma variável dicotómica, mas sim contínua e complexa. Tendo em conta o que foi previamente referido, deve haver uma consciência de que cada sociedade evolui de forma semi-autónoma, ainda que sob a influência de uma determinada cultura geral, dando origem a uma variabilidade inter e intra-grupal que se traduz num conjunto diverso de práticas e crenças (Costigan, Bardina, Cauce, Kim \& Latendresse, 2006). Isto ocorre não só entre países, mas também dentro de diferentes grupos de cada país, como resultado de diferenças nas condições sociais, políticas e/ou económicas. Na prática psicológica multicultural, é importante procurar compreender tanto as particularidades individuais como grupais e socioculturais do cliente, evitando estereótipos. 
Em países asiáticos (por exemplo Coreia e Japão, com especial ênfase neste último) imperam alguns valores distintos dos que caracterizam as culturas ocidentais europeias e norte-americana, sendo estas últimas tradicionalmente individualistas (Neto, 2003). Uma das principais diferenças culturais entre ambos os tipos de sociedade reside no facto de vigorar nos países asiáticos, tendencialmente, um modelo de sociedade mais colectivista, havendo uma primazia do grupo sobre o indivíduo. Triandis (1988, citado por Neto, 2003) caracteriza o estilo colectivista da seguinte forma:

a) Coloca ênfase nas perspectivas, necessidades e objectivos do colectivo;

b) O comportamento é função das normas e dos deveres impostos pelo colectivo;

c) Ênfase em crenças partilhadas, naquilo que o indivíduo e o colectivo partilham;

d) O comportamento social é cooperativo e altruísta em relação aos membros do endogrupo, mas indiferente e até hostil com os membros do exogrupo.

Paralelamente a este modelo de organização social, encontram-se também costumes diferentes que podem muitas vezes originar "choques culturais" no contacto entre elementos dos vários tipos de culturas, assumindo frequentemente a forma de embaraço. Apresenta-se como exemplo disto o desconforto provocado pelo contacto visual prolongado, na cultura japonesa, sendo esse tipo de contacto ocular relativamente comum em sociedades ocidentais, particularmente durante o estabelecimento de conversas (Okano, 1994). Alguns autores referem várias diferenças culturais a ter em conta, no contexto relacional, e também na prática de consulta psicológica (Sommers-Flanagan \& Sommers-Flanagan, 2004), sendo que neste último contexto se devem considerar sempre eventuais especificidades culturais.

Para Dinnel e co-autores (2002), uma das componentes fundamentais da dimensão social individualismo-colectivismo é a variável independência-interdependência: a caracterização da auto-imagem, por parte dos indivíduos, como independente ou interdependente (associando-se geralmente uma maior independência a estilos mais individualistas). Referem no entanto que a cultura de origem representa apenas um tendência geral dessa caracterização, e não uma certeza. $O$ conceito de auto-imagem e a variável a ele associado têm vindo a receber particular atenção desde os anos 80, enquanto elementos fundamentais no estudo da relação entre o indivíduo e a sociedade, tendo já sido aplicados em diferentes estudos (Gouveia, Singelis $\&$ Coelho, 2002). Kleinknecht e co-autores (1997) defendem que o traço 
independência-interdependência não é dicotómico nem culturalmente restrito, podendo um indivíduo apresentar elementos de ambas as formas de caracterização, em graus variáveis, independentemente da cultura de origem. A cultura exerce uma influência, que depois se processa de diferentes formas, em interacção com factores individuais, sociais, e até contextuais.

Estabelecendo a ligação entre a interacção de factores culturais e pessoais e o tema da ansiedade social, Gouveia (2000) salienta de forma breve o papel da variável independência-interdependência como a principal diferença entre os focos da ansiedade social tal como vivenciada nas culturas ocidental e oriental. A ligação entre esta variável e a ansiedade social é igualmente referida por outros autores (Dinnel et al., 2002; Kleinknecht et al., 1997), associando-se tendencialmente os sintomas de TKS a valores mais elevados de interdependência. Em termos genéricos, considera-se que a forma como o indivíduo constrói a sua auto-imagem será determinante para a formação das crenças deste relativamente ao que pode constituir uma ameaça à mesma, e em que grau. Como tal, os estímulos sociais que são percepcionados como mais ameaçadores são também aqueles que provavelmente originarão maior ansiedade.

\subsection{Embaraço}

É pertinente definir o que constitui o embaraço, e o que é um acto embaraçoso, definições essas que podem variar consoante a cultura de referência ${ }^{4}$. Segundo Neto (2003, p. 249), alguns autores (Buss, 1981; Schlenker \& Leary, 1982) defendem que o embaraço é geralmente visto como "uma forma de ansiedade social, intimamente relacionada com a timidez, a ansiedade em público e a vergonha", sendo as suas características comparáveis às de uma qualquer emoção. Embora o conceito de "vergonha" lhe seja frequentemente associado, a distinção entre estas duas emoções auto-conscientes é geralmente dificultada pela ausência de consenso científico (Tangney, Miller, Flicker \& Barlow, 1996). De acordo com uma das diferentes perspectivas, aqui adoptada, é argumentado que o embaraço resulta de uma percepção de violação pública de costumes sociais (de natureza não-moral), ao passo que a vergonha resulta da percepção de violação de uma conduta moral, podendo esta ocorrer tanto em contexto público como privado (Harris, 2003). Como tal, o embaraço é definido como sendo derivado de um acto público, percebido como socialmente reprovável (por oposto a

\footnotetext{
${ }^{4}$ Um exemplo disto ocorre relativamente ao uso de vestuário, ou ausência deste, em contexto social (Titiev, 2002): será pouco provável que um indivíduo pertencente a uma sociedade onde os indivíduos andem parcialmente sem vestuário possa ficar embaraçado por ser surpreendido com o corpo exposto.
} 
moralmente errado), tornando-se assim um conceito mais relevante no âmbito desta discussão.

Edelman (1985 e 1987, citado por Neto, 2003) apresenta um modelo para a explicação da experiência de embaraço, propondo que:

a) O processo [de embaraço] é activado por uma preocupação com as regras sociais e o desejo de evitar uma perda de aprovação social. Uma discrepância indesejada entre o comportamento que se está a desenrolar e o padrão estabelecido é passível de levar a focar a atenção no aspecto público da auto-imagem, com uma activação consequente de preocupação acerca da sua própria identidade e da geração de afecto negativo;

b) O indivíduo usa, então, informação dos seus próprios comportamentos expressivos associados a este afecto negativo, tais como pistas viscerais e memória de episódios passados de quebra de regras, para categorizar a experiência como embaraço;

c) Como é difícil esconder o embaraço, pode ser adoptado um número de estratégias remediativas para recuperar a percepção de aprovação social perdida e repor a imagem pública do actor.

O embaraço é geralmente definido como a vivência de um estado emocional desagradável, no decurso de um acto ou condição socialmente inaceitável, testemunhada por, ou revelada a, outras pessoas. Tratando-se de uma emoção mediada pela aquisição de uma consciência de si próprio e pela aprendizagem de normas sociais, o embaraço não é caracterizado como uma emoção primária (as quais são consideradas inatas, não necessitando de ser precedidas por competências cognitivas específicas), mas sim como uma emoção auto-consciente secundária (Lewis, Sullivan Stanger \& Weiss, 1998). Segundo estes autores, o embaraço surge por volta dos dois anos de idade, tal como a empatia e a inveja, constituindo este grupo um primeiro tipo de emoções auto-conscientes. Reforçando a diferenciação entre os conceitos de embaraço e vergonha, os autores acrescentam que um segundo tipo de emoções auto-conscientes surge após estas primeiras, caracterizado por uma natureza auto-avaliativa e requerendo não só uma maior capacidade cognitiva como também a aprendizagem de padrões e regras de conduta, incluindo-se nesta segunda categoria a vergonha, o orgulho e a culpa.

Com base no conceito de embaraço discutido, é previsível que alguns dos estímulos considerados embaraçosos (e geradores de ansiedade social) na cultura ocidental não o sejam noutras culturas, da mesma forma que o que é "socialmente reprovável" numa sociedade pode não o ser noutra. Consequentemente, importa compreender a experiência de embaraço como uma crença por parte do indivíduo de que se encontra numa situação que acredita ser considerada socialmente reprovável (no seu contexto social de 
vida), despoletando-se os mecanismos de protecção que este tiver adquirido ao longo do seu ciclo vital (adaptativos ou desadaptativos). Mesmo dentro duma cultura específica, as situações nas quais essa crença pode surgir não são uniformes, sendo influenciadas por factores individuais. Um indivíduo pode, por exemplo, encontrar-se numa situação socialmente reprovável e, ao contrário das pessoas que o rodeiam, não a considerar embaraçosa ou sequer reprovável, e vice-versa.

Para uma melhor compreensão da experiência de embaraço, esta pode ser enquadrada numa perspectiva evolutiva: estando a sobrevivência e subsistência do Ser Humano estreitamente ligadas aos seus pares, através de uma interacção produtiva em contexto social, a imagem social do indivíduo assume-se como algo que deve idealmente ser protegido de afectos negativos alheios, de acordo com as regras específicas da sociedade envolvente, no sentido de manter viáveis e funcionais as relações significativas. A esta imagem pública estão frequentemente associadas questões de relacionamento, integração e estatuto (Beck \& Emery, 1985), ocorrendo a experiência de embaraço quando o indivíduo de alguma forma valoriza o julgamento da pessoa ou pessoas que testemunham a violação da norma social. Consequentemente, perante situações de percepção de ameaça a essa mesma imagem, a emoção auto-consciente de embaraço surge como motivadora de acção no sentido de lidar com essa ameaça, de forma a repor o cumprimento da norma social em causa e procurar recuperar a percepção de aprovação social. Para Gouveia, Singelis, Guerra, Santos e Vasconcelos (2005), o embaraço pode ser considerado como tendo a função de actuar como um mecanismo emocional que permite às pessoas manter a estabilidade das suas comunidades e das interacções sociais quotidianas.

\subsection{Interacção entre as variáveis apresentadas}

Kleinknecht e co-autores (1997), no seu estudo, procuraram investigar a interacção entre as variáveis apresentadas, tendo aplicado escalas de ansiedade social e de taijin-kyofu-sho em conjunto com uma escala de avaliação da dimensão independência-interdependência, em amostras não-clínicas estudantis (em contexto americano e japonês). Nas suas conclusões, referem que devido ao facto de cada país definir a sua síndrome de acordo com o modelo geral de sociedade (como individualista ou como colectivista), os seus clínicos teriam menos tendência para solicitar aos seus pacientes relatos de sintomas do tipo oposto, ou seja, relatos de sintomas de ansiedade social protectores do grupo em sociedades individualistas, e protectores do indivíduo em sociedades colectivistas. Acrescentam que apesar disto, sintomas de ambas as variantes de ansiedade social existem de facto nas duas culturas estudadas, devendo os profissionais alargar a sua perspectiva sobre a ansiedade social. Concluíram também que uma auto-imagem caracterizada por valores mais 
elevados de independência funciona geralmente como um factor de resiliência face a ansiedade social, podendo resultar numa menor interpretação do julgamento negativo de outras pessoas como uma ameaça. Para além disso, afirmam que, da mesma forma que o traço independência-interdependência não é dicotómico (podendo um indivíduo de cada uma das culturas apresentar elementos de ambas as formas de caracterização, em graus variáveis), também cada indivíduo pode apresentar diferentes graus de cada tipo de vivência da ansiedade social, individual ou grupal. Finalmente, não tendo sido observada na amostra de população japonesa uma relação significativa entre valores mais elevados de interdependência e os resultados da escala de TKS, ao contrário do que era esperado, os autores levantam a hipótese de que a aplicação das escalas a uma amostra clínica de TKS permitiria um melhor estudo de como essas variáveis se relacionam.

Por sua vez, Gouveia e co-autores (2005) procuraram observar a relação entre as duas formas de caracterização da auto-imagem e o sentimento de embaraço (aí definido como "constrangimento"), vivenciado em diversas circunstâncias sociais. Para tal, utilizaram uma escala de constrangimento e uma escala de auto-imagem, aplicada a uma amostra brasileira composta por uma maioria de estudantes universitários e do ensino secundário. Entre os resultados obtidos, destaca-se a existência de uma correlação significativa negativa entre o embaraço e valores mais acentuados de independência, e uma correlação significativa positiva entre o embaraço e valores mais acentuados de interdependência. Este resultado veio ao encontro da hipótese inicialmente levantada pelos autores, formulada com base em estudos prévios realizados numa outra população (estudantes da Universidade do Havai). Como tal, concluíram que uma auto-imagem tendencialmente interdependente actua como um melhor preditor da experiência de embaraço, ao passo que os indivíduos com valores mais elevados de independência tenderam a demonstrar menos embaraço.

\section{A problemática dos sintomas secundários e de estigmas sociais}

Analisemos os possíveis efeitos deste tipo de ansiedade a nível pessoal e social. Muitas vezes, a ansiedade social nas suas diferentes formas pode levar ao desenvolvimento de consequências e sintomas secundários, tais como dificuldades de desempenho e integração sociais, depressão, e até o isolamento social. Este isolamento constitui um extremo do evitamento das situações sociais anxiogéneas, sendo para muitos indivíduos uma fuga subjectiva às dificuldades de integração social. No mínimo, existe uma redução da qualidade de vida, podendo a vivência da ansiedade provocar no indivíduo níveis de sofrimento consideráveis. 
No contexto da cultura japonesa onde, como discutido, predominam valores colectivistas e onde se verificam acentuadas pressões dos sistemas educativo e profissional sobre o indivíduo, a ansiedade de origem social pode actuar como um dos factores facilitadores de um outro fenómeno conhecido como "hikikomori" (significando literalmente "reclusão / estar confinado") 5 . Este quadro é referente a indivíduos, geralmente jovens, entre os 15 e os 26 anos, que se isolam no quarto durante um período superior a seis meses (chegando nos casos mais extremos a anos ou mesmo décadas), com evidentes prejuízos para as suas capacidades já diminutas de socialização e em termos de custos familiares e sociais. Origina-se um isolamento social agudo que, segundo estimativas, afecta entre 100.000 a 320.000 adolescentes japoneses, de acordo com as perspectivas mais conservadoras (Jones, 2006). Segundo a mesma referênca, ao passo que na década de 80 estas ocorrências eram ainda interpretadas como algo pontual e consequentemente ignorado pelos sistemas de saúde do país, restringido a jovens que se refugiavam no quarto a ver televisão e a jogar jogos de vídeo, tornou-se nas décadas seguintes uma crescente preocupação nacional. No entanto, no Japão esta problemática não é considerada pela maioria da população como uma perturbação mental, mas sim como um problema familiar (Furlong, 2008; Rees, 2002a; Rees, 2002b). Neste contexto cultural, é geralmente atribuído ao estabelecimento de uma relação de dependência entre mãe e filho/filha (estando este fenómeno particularmente associado a primogénitos, com maior predominância de jovens do sexo masculino), e às fortes pressões do sistema educativo e profissional que se fazem sentir, tanto sobre os pais como sobre os filhos. Dada esta visão não-medicalizada, que atribui responsabilidades à família, existe ainda um forte estigma social, em relação tanto aos indivíduos "hikikomori" como aos seus familiares, devido em grande parte à ênfase colocada no colectivo enquanto unidade funcional. Este estigma suscita muitas vezes dificuldades de re-integração social para os indivíduos, surgindo actualmente associações destinadas exclusivamente à tentativa de facilitação gradual desse processo de re-inserção social, de outra forma quase impossível. O medo de revelação pública de uma situação destas no seio familiar origina também frequentemente uma maior passividade por parte dos pais, resistência em procurar ajuda especializada exterior e, consequentemente, um maior prolongamento do problema.

$\mathrm{O}$ isolamento social de adolescentes, no decurso de uma maior dificuldade de integração social e/ou de depressão, é verificável em várias partes do mundo, embora também com as respectivas variações culturais. Nas

\footnotetext{
${ }^{5}$ Sendo este tema ainda pouco estudado fora do Japão, as principais fontes utilizadas para recolher informação consistiram em artigos e vídeos de divulgação (não-científicos) produzidos por correspondentes de meios de comunicação da BBC e New York Times (cf. Jones, 2006; Rees, 2002a; Rees, 2002b).
} 
sociedades ocidentais, apesar de o estigma relativo a dificuldades de integração e desempenho social ser menos acentuado, é igualmente sentido de diferentes formas. Os indivíduos com elevada ansiedade social, particularmente do tipo generalizado, tendem a ter uma menor possibilidade de acesso a oportunidades sociais e profissionais, devido ao evitamento de situações de interacção social. Baseando-se a sociedade actual cada vez mais num modelo de competição, onde as capacidades de relacionamento interpessoal, comunicação e dinamismo são cada vez mais valorizadas (Okano, 1994), verifica-se uma óbvia desvantagem a nível profissional que, por sua vez, origina também uma maior incerteza e consequente pressão psicológica sobre os indivíduos. Está ainda por determinar de forma científica e objectiva a sensibilidade das entidades empregadoras e da sociedade em geral em relação a indivíduos com problemáticas de ansiedade e/ou isolamento social. O isolamento social, por sua vez, é visto como um problema médico, sendo a família vista como um factor de risco ou de protecção, e não necessariamente a causa central (dependendo dos casos). Uma questão fundamental se coloca: poderão as crescentes pressões educacionais e profissionais, sentidas também nas sociedades ocidentais ${ }^{6}$, fazer sentir-se de igual forma no contexto de uma cultura tendencialmente individualista como a ocidental?

Evidenciam-se assim outros pontos de contacto e distinções em vivências psicopatológicas decorrentes de variações sociais ou culturais. Por este motivo, torna-se necessária uma maior atenção aos factores socioculturais que originam e promovem a continuidade de vivências psicopatológicas, de forma a procurar compreender e atenuar os seus efeitos. Apesar de alguns fenómenos terem uma clara marca cultural, fundamental no seu surgimento e manutenção, possuem também outros factores determinantes que não devem ser de forma alguma ignorados, de ordem social, contextual, e individual, porventura comuns à vivência humana.

\section{Discussão e Conclusão}

Na Figura 1 (em anexo) procura-se ilustrar as principais fontes de influência de aspectos socioculturais na manifestação da ansiedade social. Pretende-se representar também a importância dos factores individuais, que assumem um papel central relativamente aos outros elementos, ao influenciarem e serem influenciados pela aprendizagem social dos indivíduos.

Ao estudar a ansiedade social e suas variantes é possível observar os pontos de contacto entre elas, embora alguns aspectos da actual visão da

\footnotetext{
${ }^{6}$ Derivadas, em parte, de um aumento das taxas de desemprego e da precariedade do trabalho (Azevedo, 1999), pelo menos no que respeita à sociedade portuguesa.
} 
ansiedade social e da TKS caracterizem estes quadros clínicos como algo distinto e não como quadros semelhantes com determinantes culturais, não se tendo muitas vezes em conta os factores que os originam. A ênfase é geralmente colocada nos sintomas, por oposição à origem dos mesmos, o que leva a uma classificação distinta de problemas que se podem considerar equiparáveis, no sentido de representarem diferentes formas do mesmo quadro. No entanto, tem havido um movimento crescente no sentido de expandir a forma como se conceptualiza a ansiedade social, perante a tentativa de compreensão dos factores culturais e pessoais que a determinam.

Seria útil a introdução de uma variável de clarificação, destinada a fazer a ponte entre as duas formas de ansiedade social discutidas ao ter em conta aspectos sociais. A distinção entre dois tipos de manifestação do quadro geral de ansiedade social (que podem também manifestar-se simultaneamente em diferentes graus), caracterizados pelo foco subjectivo atribuído pelo indivíduo à sua vivência ansiosa: uma ansiedade social auto-centrada (estando o foco ansioso na imagem social do próprio indivíduo), e uma ansiedade social hetero-centrada (estando o foco da ansiedade nas outras pessoas e na imagem social dos grupos de pertença, tais como a família, elementos do local trabalho, ou outras figuras significativas). A primeira, geralmente associada a uma auto-imagem caracterizada por valores mais elevados de independência, e a segunda associada a níveis mais elevados de interdependência. Há no entanto uma pequena ressalva: sendo os valores mais altos de independência considerados como potencialmente protectores em relação à manifestação de sintomas de ansiedade (Kleinknecht et al., 1997), gerando no indivíduo uma menor percepção do julgamento negativos dos outros enquanto ameaça, como se justifica a associação desses mesmos valores de independência à ansiedade auto-centrada? A explicação pode estar relacionada com o facto de que, por mais elevado que seja o grau de independência de um indivíduo, nunca é colocada em causa a sua necessidade geral de integração social, mas sim a forma como esta se processa em termos de qualidade e quantidade.

$\mathrm{Na}$ prática clínica é importante relacionar os diversos factores e as suas interacções, devendo adoptar-se uma visão do indivíduo enquanto Ser biopsicosocial complexo que influencia e é influenciado pelo seu meio, e da ansiedade social enquanto quadro clínico multi-determinado. Exploramos aqui a importância de dois factores predominantemente socioculturais relevantes: a dimensão independência-interdependência e o conceito de "socialmente reprovável" como origem da experiência de embaraço. Tal como Kleinknecht e co-autores (1997) sugerem, é fundamental que os investigadores e psicoterapeutas expandam as suas definições de ansiedade social, sendo essencial que estas incluam o papel de influências culturais. Deriva daí a possibilidade de que, quando tal não ocorre, possam ser des- 
considerados sintomas que não se enquadram na definição textual culturalmente restrita, podendo assim originar-se avaliações e intervenções não totalmente condizentes com as reais necessidades do cliente.

Uma vez que um dos modelos que tem vindo a ser mais utilizado na intervenção nesta problemática tem sido o cognitivo-comportamental (Butler, 1999; Gouveia, 2000), é fundamental uma apropriada exploração do tipo, grau e foco de ansiedade exibidos pelo indivíduo para melhor compreender os seus padrões de funcionamento, permitindo assim uma adequação correcta da reestruturação cognitiva e estratégias comportamentais a aplicar. Tomando-se como um dos focos possíveis para a disputa racional o conceito subjectivo que cada cliente tem do que constitui um episódio embaraçoso (socialmente reprovável) para si ou outros, e quais as suas consequências, é importante perceber, juntamente com este, qual considera ser o comportamento esperado de si enquanto indivíduo e enquanto membro de um grupo específico, trabalhando no sentido de tornar adaptativas eventuais crenças desadaptativas ou de corrigir eventuais défices em termos de competências sociais e de gestão de ansiedade. De igual relevância é procurar avaliar que modalidade de terapia, individual ou em grupo, se adequa melhor a cada caso. Estes factores assumem particular relevância na prática de consulta psicológica em contextos multi-culturais (Sommers-Flanagan \& Sommers-Flanagan, 2004).

Procurou-se valorizar e incentivar um olhar antropológico-cultural sobre aspectos psicológicos e sobre a própria intervenção psicológica, promovendo uma visão abrangente e trans-disciplinar do Ser Humano e suas vivências, por oposição a uma abordagem centrada no campo individual e somático (que muitas vezes ignora factores sociais e/ou culturais). Para Kirmayer (2001), a investigação psiquiátrica convencional não tem sido totalmente adequada no que concerne à exploração do significado cultural do sofrimento, devido ao facto de tentar reduzir a complexidade das narrativas da doença a uma lista de sintomas e sinais significativos. Este autor salienta, no entanto, que se tem verificado um número crescente de investigações epidemiológicas que recorrem à Antropologia Cultural (também referenciada como Etnografia, Antropologia Social, ou Etnologia), para complementar as suas observações, no sentido de identificar variações culturais fundamentais. A aculturação, processo através do qual as reacções individuais se vão ajustando aos padrões de uma sociedade, e a aprendizagem social, processo através da qual a cultura é transmitida aos indivíduos, assumem-se aqui como factores centrais na forma geral de manifestação de algumas formas de sofrimento, ideia reforçada no DSM-IV-TR: "podem ser encontradas apresentações conformes com a maioria das categorias do DSM-IV praticamente por todo o mundo, sendo os sintomas particulares, a evolução e a resposta social muitas vezes influenciados por factores cultu- 
rais locais" (APA, 2002, p. 898). O papel da aculturação, por sua vez, está ainda por determinar objectivamente.

Teve-se aqui como objectivo central fazer uma revisão de conceitos relativos aos efeitos da cultura sobre variáveis pessoais com relação à problemática da ansiedade social, defendendo-se a ideia de que a ansiedade social é um fenómeno relativamente universal no que respeita à vida em sociedade (tal como defendido por Kleinknecht et al., 1997) devido em grande parte a questões hierárquicas e de integração, havendo no entanto variações decorrentes da interacção ente aspectos culturais e aspectos individuais. Procurou-se fazer uma breve caracterização de quais são essas variações, usando como exemplo central a comparação entre culturas ocidentais (europeia e americana) e culturas orientais (asiáticas, particularmente a japonesa), e determinar quais as suas implicações em termos de conceptualização e de intervenção.

A vida em sociedade e a integração nesta, por muita mínima que seja, sempre foram condições sine qua non para a sobrevivência / subsistência dos indivíduos que a ela pertencem, condicionando a vida pessoal e profissional de cada um. Citando Titiev (2002, p. 19), “(...) não nos podemos comportar como seres humanos a não ser que interactuemos, pelo menos ocasionalmente, com outros membros da nossa espécie". Quando os indivíduos falham nessa tentativa de integração, incorrem em elevados níveis de sofrimento psicológico e/ou encontram por vezes um refúgio subjectivo no evitamento ou isolamento social (entre outras alternativas não abordadas neste artigo), com os respectivos custos pessoais, grupais e sociais que isso acarreta. Tendo em conta a quantidade de pessoas afectadas pela ansiedade social desadaptativa nas suas mais diversas formas e o efeito desta na qualidade de vida desses indivíduos, torna-se essencial uma especial atenção para este quadro na sua generalidade, juntamente com a análise de elementos culturais, sociais e individuais (e seus significados), por oposto à focalização exclusiva em factores individuais e somáticos.

\section{Referências}

American Psychiatric Association [APA] (2002). Manual de Diagnóstico e Estatística das Perturbações Mentais $-4^{a}$ Edição - Texto Revisto. Lisboa: Climepsi Editores.

Azevedo, J. (1999). Voos de Borboleta: Escola, Trabalho e Profissão. Porto: Asa Editores.

Beck, A. \& Emery, G. (1985). Anxiety Disorders and Phobias: a Cognitive Perspective. New York: Basic Books.

Butler, G. (1999). Overcoming Social Anxiety and Shyness. London: Robinson Publishing Ltd. 
Costigan, C., Bardina, P., Cauce, A., Kim, G., \& Latendresse, S. (2006). Inter- and Intra-Group Variability in Perceptions of Behavior among Asian Americans and European Americans. Cultural Diversity and Ethnic Minority Psychology, 12 (4), 710-724.

Dinnel, D., Kleinknecht, R., \& Tanaka-Matsumi, J. (2002). A Cross-Cultural Comparison of Social Phobia Symptoms. Journal of Psychopathology and Behavioral Assessment, 24 (2), 75-84.

Furlong, A. (2008). The Japanese Hikikomori Phenomenon: Acute Social Withdrawal among Young People. Sociological Review, 56 (2), 309-325.

Gielens, U., Draguns, J., \& Fish, J. (2008). Principles of Multicultural Counseling and Therapy. New York: Routledge.

Gouveia, J. (2000). Ansiedade Social: da Timidez à Fobia Social. Coimbra: Quarteto Editora.

Gouveia, V., Singelis, T., \& Coelho, J. (2002). Escala de Auto-Imagem: Comprovação da sua Estrutura Fatorial. Avaliação Psicológica, 1, 49-59.

Gouveia, V., Singelis, T., Guerra, V., Santos, W., \& Vasconcelos, T. (2005). Auto-Imagem e Sentimento de Constrangimento. Psico, 36 (3), 231-241.

Harris, N. (2003). Reassessing the dimensionality of the moral emotions. British Journal of Psychology, 94 (4), 457-473.

Heimberg, R., \& Becker, R. (2002). Cognitive-Behavioral Group Therapy for Social Phobia. New York: The Guilford Press.

Lewis, M., Sullivan, M., Stanger, C., \& Weiss, M. (1998). Self-Development and Self-Conscious Emotions. In J. Jenkins, K. Oatley \& N. Stein (Eds.). Human Emotions: A Reader (pp. 158-167). Massachusetts: Wiley-Blackwell.

Jones, M. (2006, 15 de Janeiro). Shutting Themselves In. The New York Times - on the Web (artigo retirado a 15/01/2006, de http://www.nytimes.com).

Kirmayer, L. (2001). Cultural Variations in the Clinical Presentation of Depression and Anxiety: Implications for Diagnosis and Treatment. Journal of Clinical Psychiatry, 62 (13), 22-30.

Kleinknecht, R., Dinnel, D., Kleinknecht, E., Himura, N., \& Harada, N. (1997). Cultural Factors in Social Anxiety: A Comparison of Social Phobia Symptom and Taijin Kyofusho. Journal of Anxiety Disorders, 11, 157-177.

Marks, I., \& Gelder, M. (1966). Different Ages of Onset in Varieties of Phobia. American Journal of Psychiatry, 123, 218-221.

McNally, R., Cassiday, K., \& Calamari, J. (1990). Taijin-Kyofu-Sho in a Black American Woman: Behavioral Treatment of a "Culture-Bound" Anxiety Disorder. Journal of Anxiety Disorders, 4, 83-87.

Neto, F. (2003). Estudos de Psicologia Intercultural: Nós e os Outros (2a Edição). Lisboa: Fundação Calouste Gulbenkian.

Okano, K. (1994). Shame and Social Phobia: a Trans-Cultural Viewpoint. Bulletin of the Menninger Clinic, 58 (3), 323-338.

Okazaki, S. (2003). Expressions of Social Anxiety in Asian-Americans. Psychiatric Times, 20, nr.10. 
Organização Mundial de Saúde [OMS] (1993). Classificação de Transtornos Mentais e de Comportamento da CID-10. Porto Alegre: Artes Médicas.

Pryse-Phillips, W. (1971). An Olfactory Reference Syndrome. Acta Psychiatry Scandinavia, 47, 484-509.

Rees, P. (correspondente) (2002a). The Mystery of the Missing Million [documentário]. Reino Unido: British Broadcasting Corporation (BBC Two).

Rees, P. (2002b, 20 de Novembro). Japan: The Missing Million. BBC News World Edition (artigo retirado a 25/12/2005, de http://news.bbc.co.uk/).

Seedat, S., \& Nagata, T. (2004). Cross-Cultural Aspects of Social Anxiety Disorder. In B. Bandelow \& D. Stein (Eds.). Social Anxiety Disorder (pp. 104-116). New York: Marcel Dekker Inc.

Sommers-Flanagan, J. \& Sommers-Flanagan, R. (2004). Counseling and Psychoterapy Theories in Context and Practice. New Jersey: John Wiley \& Sons.

Suzuki, K., Takei, N., Kawai, M., Minabe, Y., \& Mori, N. (2003). Is Taijin Kyofusho a Culture-Bound Syndrome?. American Journal of Psychiatry, 160 (7), 1358.

Tangney, J., Miller, R., Flicker, L., \& Barlow, D. (1996). Are shame, guilt, and embarrassment distinct emotions?. Journal of Personality and Social Psychology, 70 (6), 1256-1269.

Takahashi, T. (1989). Social Phobia Syndrome in Japan. Comprehensive Psychiatry, $30,45-52$.

Titiev, M. (2002). Introdução à Antropologia Cultural ( $\left(9^{a}\right.$ Edição). Lisboa: Fundação Calouste Gulbenkian.

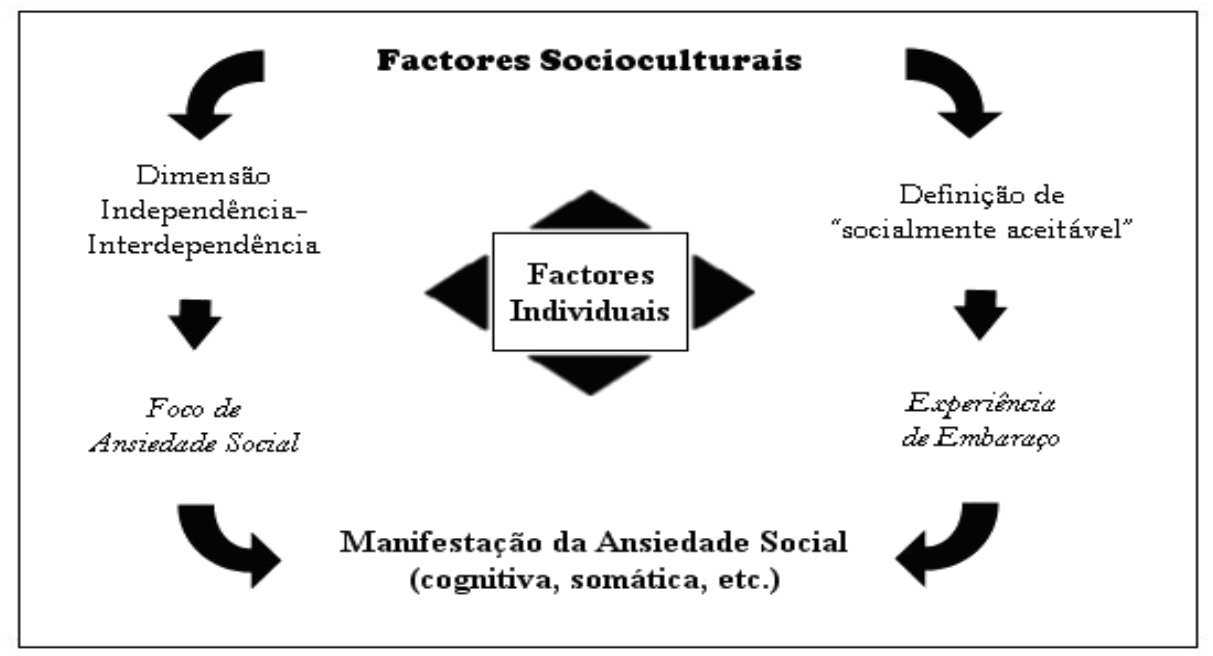

Figura 1 - Influência de Factores Socioculturais na Manifestação da Ansiedade Social. 